\title{
Electron-positron momentum distributions and positron lifetime in semiconductors in the generalized gradient approximation
}

\author{
B. K. Panda, W. LiMing, S. Fung, and C. D. Beling \\ Department of Physics, The University of Hong Kong, Hong Kong, China \\ (Received 19 December 1996; revised manuscript received 17 April 1997)
}

\begin{abstract}
The positron annihilation characteristics have been calculated taking the electron-positron correlation in the generalized gradient approximation (GGA). The calculated electron-positron momentum distributions in $\mathrm{Si}$ along the [110] direction in the GGA scheme agree very well with the experiment. The comparison of anisotropies of the momentum distributions along different crystal directions with the theory shows that only the GGA scheme gives the exact values. The enhancement factor for the valence electrons in the electronpositron momentum density is found to be weakly dependent on the momentum. The positron lifetimes in group IV, III-V, and II-VI semiconductors agree very well with the previous calculations and the experiment. [S0163-1829(97)05936-5]
\end{abstract}

\section{INTRODUCTION}

Positron annihilation techniques together with theoretical calculation give valuable information about the electronic structure of bulk and defects in semiconductors. ${ }^{1}$ In particular, the positron lifetime measurement technique offers a unique method to distinguish between different types of samples and samples with and without defects. Being an integrated quantity, however, the positron lifetime offers no detail of the electronic structure of the material. The angular correlation of annihilation radiation measurement yields detailed momentum distributions of the annihilating positronelectron pairs and as such presents a greater challenge to the theory. ${ }^{1}$

The theoretical positron annihilation calculation requires an accurate calculation of the electron and positron states in semiconductors. ${ }^{2}$ In the density-functional theory the ground-state property of a system of noninteracting particles is determined by its charge density. ${ }^{3}$ All many-body effects are taken in the exchange-correlation potential $V_{\mathrm{xc}}$. The electron states are the self-consistent solution of the KohnSham equations. The positron state in a system of electrons also depends strongly on the electron charge density. ${ }^{2}$ The attractive positron-electron Coulomb potential is calculated using the electron charge density. The thermalized positron attracts the surrounding electrons so that the electron density is increased at the site of the positron, which contributes a positron-electron correlation potential and an enhancement factor. The positron-electron correlation potential and the enhancement factor are determined from the electron charge density. The method of calculation that solves electron and positron states in a solid according to the above scheme is called the two-component density-functional theory (TCDFT). ${ }^{4,5}$ With one positron in a many-electron system the theory is remarkably simple because both positronpositron exchange interaction and the effect of the positron charge density on the electron states are negligibly small.

Rojas, Barbiellini, and Jarlborg have tested the effect of the positron-electron correlation potential on the positron lifetime calculation in diamond, Si, and Ge. ${ }^{6}$ Surprisingly, they found that the theory, when not taking the correlation potential into account, agreed very well with experiment, but that the annihilation rate was overestimated when the positron-electron correlation potential and the enhancement factor were calculated in the local-density approximation (LDA). The strength of the correlation potential and the enhancement factor have been reduced in two ways. Firstly, the screening is reduced by using a parameter based either on the band gap or the high-frequency dielectric constant in a semiempirical approach. ${ }^{7,8}$ This is justified because the positron screening in semiconductors is weaker than that in a homogeneous electron gas due to the band gap. Secondly, the core enhancement factor is calculated taking a constant enhancement factor. This is also justified because the core electrons are tightly bound to the nucleus and are not perturbed by the presence of the positron as the positron is repelled by the positive ion-core potential. Jensen has shown that in metals the artificial separation of the valence and core annihilation rates is not necessary and the lifetime calculated using the total electron density in the LDA agrees very well with the experiment. ${ }^{9}$ Recently Barbiellini et al. have shown that the previous form of the parameterized contact potential based on the LDA by Boronski and Nieminen ${ }^{5}$ is not correct and they have given a new form of the contact potential. ${ }^{10}$ The agreement between the theory and experiment was found to be poor using this new form of the LDA contact potential leading Barbiellini et al. to formulate a generalized gradient approximation (GGA) correction on the positron correlation potential and the enhancement factor. ${ }^{10,11}$ The calculated lifetimes are in good agreement with experiments in most of the solids including metals, semiconductors, insulators and high-temperature semiconductors.

Barbiellini et al. have employed the linear-muffin-tinorbital method within the atomic-spheres approximation (LMTO-ASA) to calculate the annihilation rate and the electron-positron momentum distributions. The LMTO-ASA is an all electron band structure method. ${ }^{12} \mathrm{~A}$ major motivation behind these calculations also lies in the fact that defect calculations in semiconductors are now based on the CarParrinello calculation, which utilizes the pseudopotential theory. ${ }^{13}$ The pseudopotential theory yields only valence 
wave functions, which lack the core-orthogonalization term. The core charge density is carried out separately using an atomic structure calculation. Therefore, a fresh calculation of the annihilation rate and the electron-positron momentum distributions is needed to test the validity of the pseudowave-functions. Moreover, the accuracy of the positron lifetime in a defect should be tested by first reproducing the experimental bulk lifetime.

We have presented the theory in Sec. II. The momentum distributions in $\mathrm{Si}$ are compared with the experiment in Sec. III. Our calculated positron lifetimes in elemental semiconductors such as diamond, $\mathrm{Si}$, and $\mathrm{Ge}$ and compound semiconductors such as $\mathrm{SiC}, \mathrm{GaAs}$, InP, and CdTe are compared with the previous theory in this section. We conclude in Sec. IV.

\section{THEORY}

The fact that the TCDFT with one positron in a manyelectron system requires the electron states calculation to be independent of the positron state simplifies the computational scheme. ${ }^{4,5}$ In the present work the nonlocal $a b$ initio electron-ion pseudopotentials of Bachelet, Hamann, and Schlüter are taken ${ }^{14,15}$ For CdTe we have taken the pseudopotentials of Stumpf and Scheffler. ${ }^{16}$ The practical interpolation scheme of Perdew and Zunger is used to calculate the exchange-correlation potential $V_{\mathrm{xc}} \cdot{ }^{17}$ The positron-ion potential is calculated using the frozen-core approximation. ${ }^{18}$

The positron annihilation rate in the LDA is given by ${ }^{9}$

$$
\lambda=\pi r_{0}^{2} c \int n_{-}(\mathbf{r}) n_{+}(\mathbf{r}) \gamma\left(n_{-}\right) d^{3} r,
$$

where $n_{-}(\mathbf{r})$ is the total electron density consisting of both the valence and core charge densities, $n_{+}(\mathbf{r})$ is the positron density, and the enhancement factor $\gamma$ in the LDA is given by ${ }^{10,11}$

$$
\gamma^{\mathrm{LDA}}\left(r_{s}\right)=1+1.23 r_{s}-0.0742 r_{s}^{2}+\frac{1}{6} r_{s}^{3},
$$

where $r_{s}=(3 / 4 \pi n)^{1 / 3}$. The two-photon momentum density is defined as

$$
\rho_{v}^{2 \gamma}(\mathbf{p})=\frac{\pi r_{0}^{2} c}{\Omega} \sum_{n \mathbf{k}}\left|\int e^{-i \mathbf{p} \cdot \mathbf{r}} \Psi_{n \mathbf{k}}(\mathbf{r}) \Psi_{+}(\mathbf{r}) \sqrt{\gamma\left(n_{-}\right)} d^{3} r\right|^{2} .
$$

The momentum-dependent enhancement factor for the valence electrons in the method of Daniuk, Sob, and Rubaszek $^{19}$ is given by

$$
\epsilon_{v}(\mathbf{p})=\frac{\sum_{n \mathbf{k}}\left|\int e^{-i \mathbf{p} \cdot \mathbf{r}} \Psi_{n \mathbf{k}}(\mathbf{r}) \Psi_{+}(\mathbf{r}) \sqrt{\gamma\left(n_{-}\right)} d^{3} r\right|^{2}}{\sum_{n \mathbf{k}}\left|\int e^{-i \mathbf{p} \cdot \mathbf{r}} \Psi_{n \mathbf{k}}(\mathbf{r}) \Psi_{+}(\mathbf{r}) d^{3} r\right|^{2}},
$$

Alatalo et al. have demonstrated that the inclusion of the position-dependent enhancement factor in the core momentum density calculation as in the valence case causes spurious effects at the high-momentum region. ${ }^{20}$ In order to overcome this problem, they have presented a method for the calculation of the state-dependent enhancement factor. In this method the core momentum density is represented as

$$
\rho_{c}^{2 \gamma}(\mathbf{p})=\frac{\pi r_{0}^{2} c}{\Omega} \sum_{j} \epsilon_{j}\left|\int e^{-i \mathbf{p} \cdot \mathbf{r}} \Psi_{j}(\mathbf{r}) \Psi_{+}(\mathbf{r}) d^{3} r\right|^{2},
$$

where the state-dependent enhancement factor $\epsilon_{j}$ is given by

$$
\epsilon_{j}=\frac{\int n_{j}(\mathbf{r}) n_{+}(\mathbf{r}) \gamma\left(n_{-}\right) d^{3} r}{\int n_{j}(\mathbf{r}) n_{+}(\mathbf{r}) d^{3} r} .
$$

This calculation is justified for core electrons because the enhancement factor is found to be independent of the momentum.

The one-dimensional (1D) momentum distributions along a particular direction are obtained by integrating the momentum density in two other directions:

$$
N\left(p_{z}\right)=\iint \rho^{2 \gamma}(\mathbf{p}) d p_{x} d p_{y} .
$$

Similarly, the two-dimensional (2D) momentum distributions are obtained by integrating the momentum density along a particular direction:

$$
N\left(p_{x}, p_{y}\right)=\int \rho^{2 \gamma}(\mathbf{p}) d p_{z} .
$$

The annihilation rate can also be obtained by integrating the momentum density over the entire momentum space $[\lambda$ $\left.=\int \rho^{2 \gamma}(p) d^{3} p\right]$. The annihilation rate is thus related to the $1 \mathrm{D}$ and $2 \mathrm{D}$ momentum distributions as

$$
\lambda=\int d p_{z} N\left(p_{z}\right)=\int d p_{x} d p_{y} N\left(p_{x}, p_{y}\right) .
$$

The autocorrelation function (AF) is defined as the Fourier transform of the momentum density, ${ }^{1}$

$$
B^{2 \gamma}(\mathbf{r})=\int \rho^{2 \gamma}(\mathbf{p}) e^{i \mathbf{p} \cdot \mathbf{r}} d^{3} p .
$$

It can be proved that the $\mathrm{AF}$ along a particular direction is the Fourier transform of the long-slit angular correlation data along that direction

$$
B^{2 \gamma}(z)=\int N\left(p_{z}\right) e^{i p_{z} z} d p_{z} .
$$

We can easily find that the $B_{v}^{2 \gamma}(0)=\lambda_{v}$ and $B_{c}^{2 \gamma}(0)=\lambda_{c}$.

The lowest-order gradient correction to the LDA correlation hole density is proportional to the parameter ${ }^{10,11}$

$$
g(\mathbf{r})=\frac{|\nabla n(r)|^{2}}{n^{2}(r) q_{\mathrm{TF}}^{2}} .
$$

where $q_{\mathrm{TF}}$ is the Therman-Fermi wave vector. The above scheme for calculating the annihilation rate and the electron momentum distributions is based on an all electron bandstructure calculation such as the LMTO-ASA. In the present calculation we have used the pseudopotential scheme, which 
gives only the valence wave functions and valence electron densities. The core electron density is usually evaluated from a separate atomic structure calculation and added to the valence electron density to obtain the total electron density. Louie, Froyen, and Cohen in their NLCC method have shown that the addition of a pseudocore electron density to the pseudovalence electron density gives accurate estimation of the exchange-correlation potential. ${ }^{21}$ In our case we are interested in calculating the electron-positron correlation potential taking total electron density into account as in the NLCC method. Therefore, we adopted their procedure for the calculation of the pseudocore electron density, which is given by

$$
n_{\mathrm{ps}}^{\alpha}(r)=\left\{\begin{array}{l}
A^{\alpha} B^{\alpha} j_{0}\left(B^{\alpha} r\right) \quad \text { for } r<r_{0} \\
n_{c}^{\alpha}(r) \quad \text { for } r>r_{0}
\end{array}\right.
$$

where $j_{0}$ is the spherical Bessel function of zeroth order and $n_{c}^{\alpha}$ is the true core electron density for the $\alpha$ atom. $r_{0}$ is the radius where the core charge density is 1.5 times the valence charge density. The constants $A$ and $B$ are determined by the value and the gradient of the core charge density at $r_{0}$. We obtain the Fourier components of the core charge density as

$$
n_{\mathrm{ps}}^{c}(\mathbf{G})=\frac{1}{\Omega} \sum_{\alpha} e^{-i \mathbf{G} \cdot \tau_{\alpha}} \int_{0}^{\infty} n_{\mathrm{ps}}^{\alpha}(r) j_{0}(G r) r^{2} d r
$$

The total charge density is obtained as

$$
n(\mathbf{r})=\sum_{\mathbf{G}}\left[n_{\mathrm{ps}}^{v}(\mathbf{G}) e^{i \mathbf{G} \cdot \mathbf{r}}+n_{\mathrm{ps}}^{c}(\mathbf{G}) j_{0}(G r)\right]
$$

where the core electron density is spherically averaged in the second term. The derivative of the total electron density is evaluated in the following way:

$$
\nabla n(\mathbf{r})=i \sum_{\mathbf{G}}\left[\mathbf{G} n_{\mathrm{ps}}^{v}(\mathbf{G}) e^{i \mathbf{G} \cdot \mathbf{r}}+\mathbf{G} n_{\mathrm{ps}}^{c}(\mathbf{G}) j_{0}(G r)\right] .
$$

The enhancement factor in the GGA is given as

$$
\gamma^{\mathrm{GGA}}\left(r_{s}\right)=1+\left[\gamma^{\mathrm{LDA}}\left(r_{s}\right)-1\right] e^{-\alpha g(\mathbf{r})},
$$

where $\alpha$ is an adjustable parameter taken to be $0.22 \cdot{ }^{10,11}$ For a uniform electron density distribution $g=0$ so that $\gamma^{\mathrm{GGA}}=\gamma^{\mathrm{LDA}}$. For core electrons the density varies rapidly so that $\epsilon$ approaches infinity. For this case $\gamma^{\mathrm{GGA}}=1$. The GGA corrected correlation energy is given by Barbiellini and coworkers as

$$
E^{\mathrm{GGA}}(\mathbf{r})=E_{\mathrm{EG}}(\mathbf{r}) e^{-\alpha g(\mathbf{r})},
$$

where $E_{\mathrm{EG}}$ is the correlation energy for the electron gas in LDA. ${ }^{1}$

In semiconductors the major contribution to the total annihilation rate mainly comes from the valence electrons due to a large interstitial space. The core contribution can be carried out in a separate calculation using the full core electron charge densities. We have calculated the valence annihilation rate in the similar method recently shown by Saito and Oshiyama, ${ }^{22}$

$$
\lambda_{v}=\pi r_{0}^{2} c \int n_{v}(\mathbf{r}) n_{+}(\mathbf{r}) \gamma\left(n_{v}\right) d^{3} r
$$

The core enhancement factor in the independent particle model (IPM) approximation is given by

$$
\lambda_{c}=\pi r_{0}^{2} c \int n_{c}(\mathbf{r}) n_{+}(\mathbf{r}) d \mathbf{r},
$$

where $n_{c}$ is the core charge density.

We have used a plane-wave basis set within the framework of the momentum-space formalism for calculating the electron and positron wave functions. ${ }^{23}$ The experimental lattice constants ${ }^{24}$ are used for all semiconductors. We have taken 350 reciprocal lattice vectors and two special points of Chadi and Cohen to compute the electron charge density. ${ }^{25}$ The core electron density and the frozen-core positron-ion potential is evaluated using atomic orbitals of Clementi and Roetti. ${ }^{26}$ The ground-state positron energy and wave function are obtained using 500 plane waves. The momentum density is calculated using 60 special points of Chadi and Cohen. ${ }^{25}$ The 1D angular correlation of position annihilation radiation experiment was normalized to the experimental positron annihilation rate.

\section{RESULTS AND DISCUSSIONS}

We have taken Si as a test case to study the effect of the GGA on the 1D-ACPAR calculations. We have added the valence AF's along different directions with the isotropic core AF to get the total AF's along that direction. Since the value of the AF's at $r=0$ in a particular method represents the annihilation rate in that method, we could easily check the correctness of our calculation. We have then Fourier transformed the AF's to obtain the momentum distributions along different directions. The experimental long-slit ACPAR data are normalized to obtain the measured annihilation rate $\left(4.6296 \mathrm{~ns}^{-1}\right)$. Fujiwara and Hyodo had normalized the theory based on IPM calculation for the valence electrons and the experiment to the same area and observed the enhancement and suppression effects. ${ }^{27}$ LiMing et al. have recently shown that the momentum distributions for the valence electrons calculated using IPM, LDA, and GGA methods differ by a constant factor. ${ }^{28}$ In other words, the momentum distributions for the valence electrons in these three methods become identical if they are normalized to the experiment. However, we have added the core momentum distributions with the valence momentum distributions and normalized our theory to the experiment in all methods. In this way we will not suffer from comparing different theories with experiment because we have got rid of the constant factor arising in the normalization of the valence electron distributions. The magnitude of momentum distributions will clearly separate the enhancement and suppression factors.

The momentum distribution along the [110] direction is shown in Fig. 1. We find that the momentum distributions calculated in the LDA and GGA methods agree very well with the experiment whereas the calculation based on the IPM method shows a marked deviation from the experiment. The IPM results are higher than the theory in the highmomentum region and the experiment falls quite sharply at the zone face compared to theory. Fujiwara and Hyodo have 


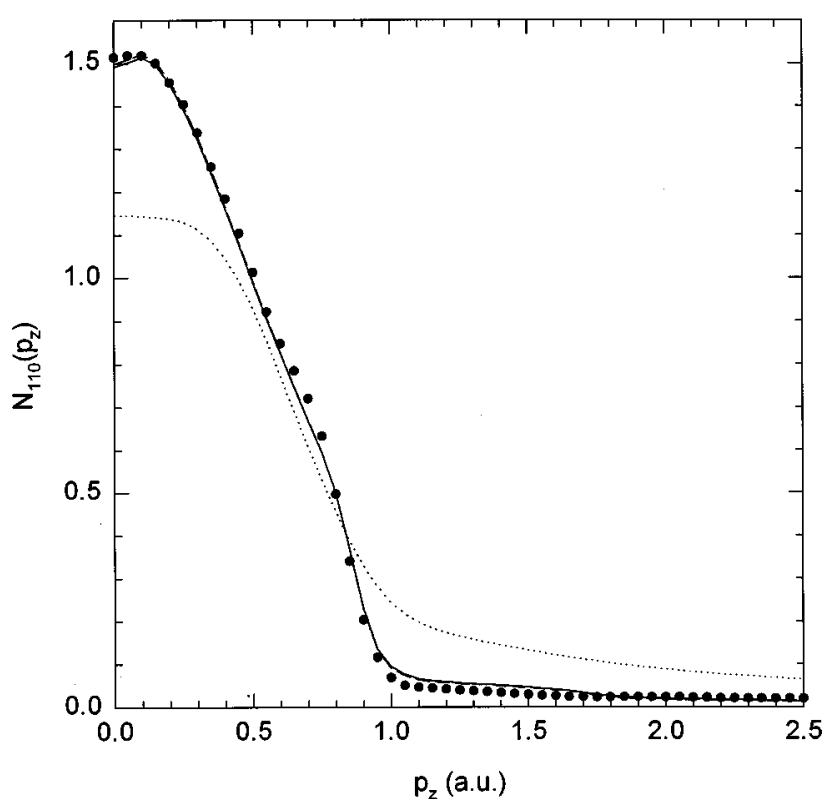

FIG. 1. Experimental and theoretical 1D ACAR along the [110] direction of Si. The experimental data of Fujiwara and Hyodo (Ref. 28) are shown by solid dots. The solid, dashed, and dotted lines correspond to the theory based on GGA, LDA, and IPM methods.

described the reduction of the experimental momentum distribution at the high-momentum region to the suppression effect and the sharp fall of the experimental data at the zone face to the enhancement effect. We have reproduced the enhancement effect in our calculation at the zone face, but in the umklapp region we have not got a good agreement. We have also not reproduced the slight hump seen at the first umklapp position. Mader et al. have shown in Al that the core-orthogonalization term in the orthogonalized planewave method reproduces the slight hump seen at the 1.3 a.u. (Ref. 29). Since our calculation is based on the pseudopotential formalism we have not reproduced good results at the umklapp region. From the lifetime analysis we will later show that our calculation does not support the idea that the core contributions are suppressed. The present formalism for the core enhancement theory does not give any suppression effect. We have not presented the comparison of the theory with experiment for other directions. A different approach has been adopted to compare our theory with experiment.

In Compton profile measurements it is a general practice to compare the experimental difference profile with the theory as the isotropic core contributions and the additive systematic experimental errors are eliminated in it. The magnitude differences between different theories as well as the multiplicative systematic errors in the experiment are eliminated by taking the percent anisotropy with respect to $N_{100}(0)$. We have adopted this procedure to compare our theory with the experiment because the accuracy of a theoretical calculations can thus be easily tested at all momenta. We have presented in the experiment in Fig. 2 the experimental and theoretical anisotropies between the [100] and [110] directions and [100] and [111] directions. The theoretical anisotropies in the GGA method matches very well with the experimental data in the low-momentum region whereas the LDA method predicts slightly higher anisotropy, and the IPM method predicts lower anisotropy. The anisotropies in

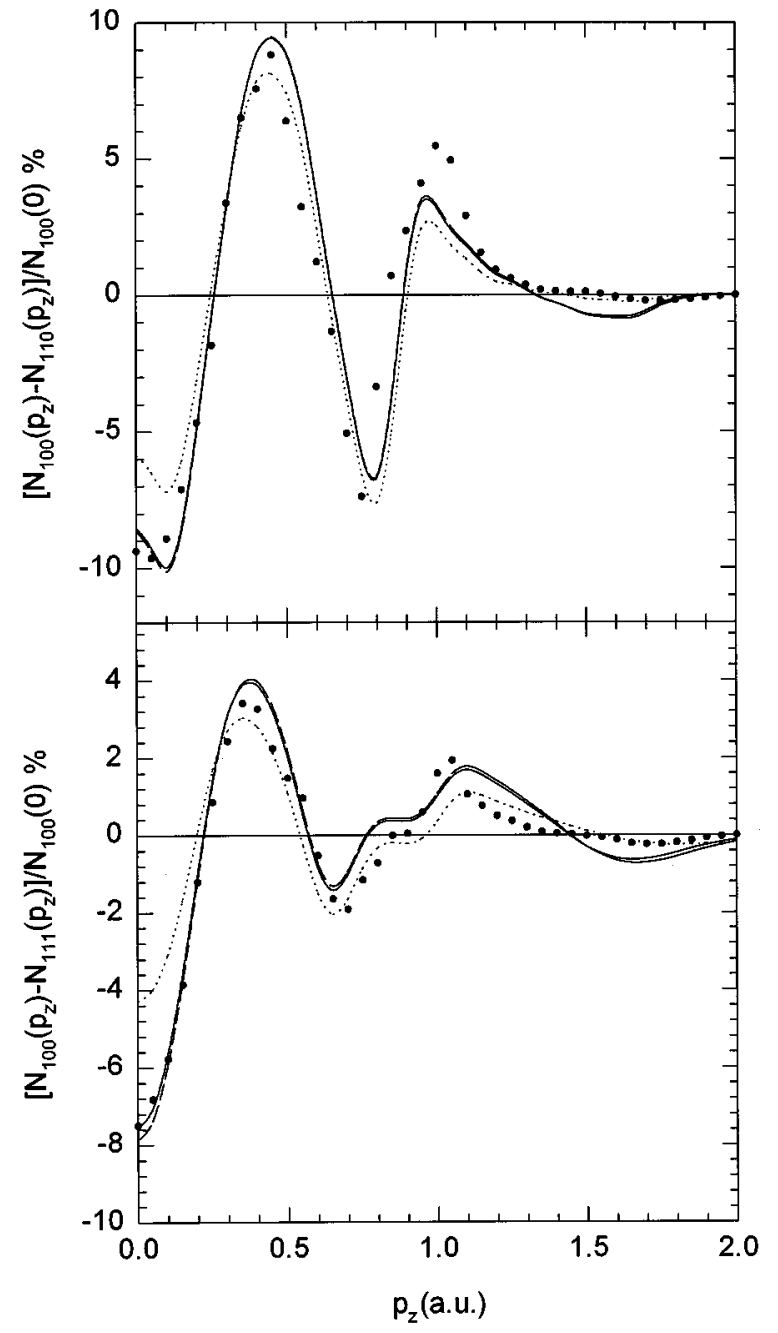

FIG. 2. Percentage anisotropy of the 1D-ACPAR data in Si. The experimental data are taken from Erskine and McGerevy (Ref. 30). The notations for the experiment, GGA, LDA, and IPM, are the same as in Fig. 1.

momentum distribution arise from the anisotropies of the electron and positron wave functions. The positron wave function calculated in the IPM method does not take the positron-electron correlation effect into account. The increase in the anisotropy in the LDA formalism shows that the GGA correction is not isotropic in nature.

The theoretical AF along the [110] direction containing both the valence and core electrons is compared with the experiment in Fig. 3. It has been shown in Compton profile experiments that the second zero crossing of the $\mathrm{AF}$ along the [110] direction shows the lattice site $\left(7.257\right.$ a.u.). ${ }^{31}$ The experimental data shows a lattice position at 7.63 a.u. The deviation of the experimental value from the actual position is due to the positron effects arising from the overlap of the positron wave function on the electron wave functions and the positron-electron enhancement factor. We have found that the theory based on the IPM calculation gives the zero position at 7.48 a.u. The inclusion of the positron-electron correlation potential in the positron-state calculation does not reproduce the experimental zero position. However, when enhancement factors based on both GGA and LDA methods are introduced into our calculation we have reproduced the 


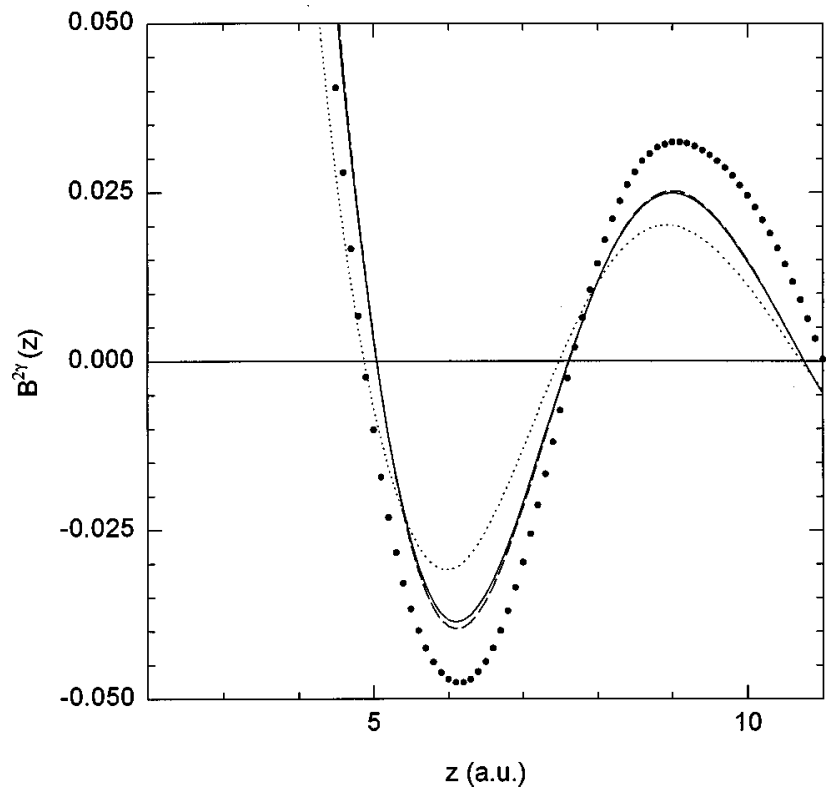

FIG. 3. Experimental and theoretical autocorrelation functions along the [110] direction of Si. The experimental data of Fujiwara and Hyodo (Ref. 27) are shown by solid dots. The notations for different theoretical approaches (GGA, LDA, and IPM) are the same as in Fig. 1.

experimental value. However, one can notice that the effect of the enhancement factor on the zero position of the AF is comparatively less than that by the positron wave function.

The valence enhancement factor $\epsilon_{v}$ along the [100], [110], and [111] directions are shown in Fig. 4. We can find that the $\epsilon_{v}$ along the [110] and [111] directions shows similar behavior. Along the [111] direction it extends to the higher momenta because the Jones zone boundary is higher along this direction. In both directions the enhancement factor increases slowly up to the zone face and then rapidly falls to zero. This shows that the $\epsilon_{v}$ is almost momentum independent, but its magnitude is different in LDA and GGA methods. However, along the [100] direction the enhancement factor shows a completely different behavior. The increasing tendency of the enhancement factor up to the the zone boundary is not observed in this case. This may be due to the fact that the positron does not see any correlation effect along this direction because there is a lot of empty space in that direction without the strong effect of the $\sigma$ and $\pi$ bonds.

The positron lifetime is calculated using both LDA and GGA schemes. We have compared the positron lifetimes for diamond, Si, and Ge with the LMTO-ASA method and the experiment in Table I. For diamond the GGA method increases the positron lifetime by 13 psec from that in the LDA method whereas in $\mathrm{Si}$ and $\mathrm{Ge}$ it is $26 \mathrm{psec}$. This is because the positron-electron correlation effect in diamond is weak as the electrons are tightly bound in it. As expected, the core annihilation rate is small in diamond and $\mathrm{Si}$, but significant in Ge. We have exactly reproduced the experimental positron lifetime in $\mathrm{Si}$ and $\mathrm{Ge}$ whereas in diamond we have got a slightly higher value. It is noteworthy to find that the positron lifetimes estimated using the total electron density as in Eq. (2.1) are found to be the same as using the valence and core annihilation rates separately in Eqs. (2.19) and (2.20). It

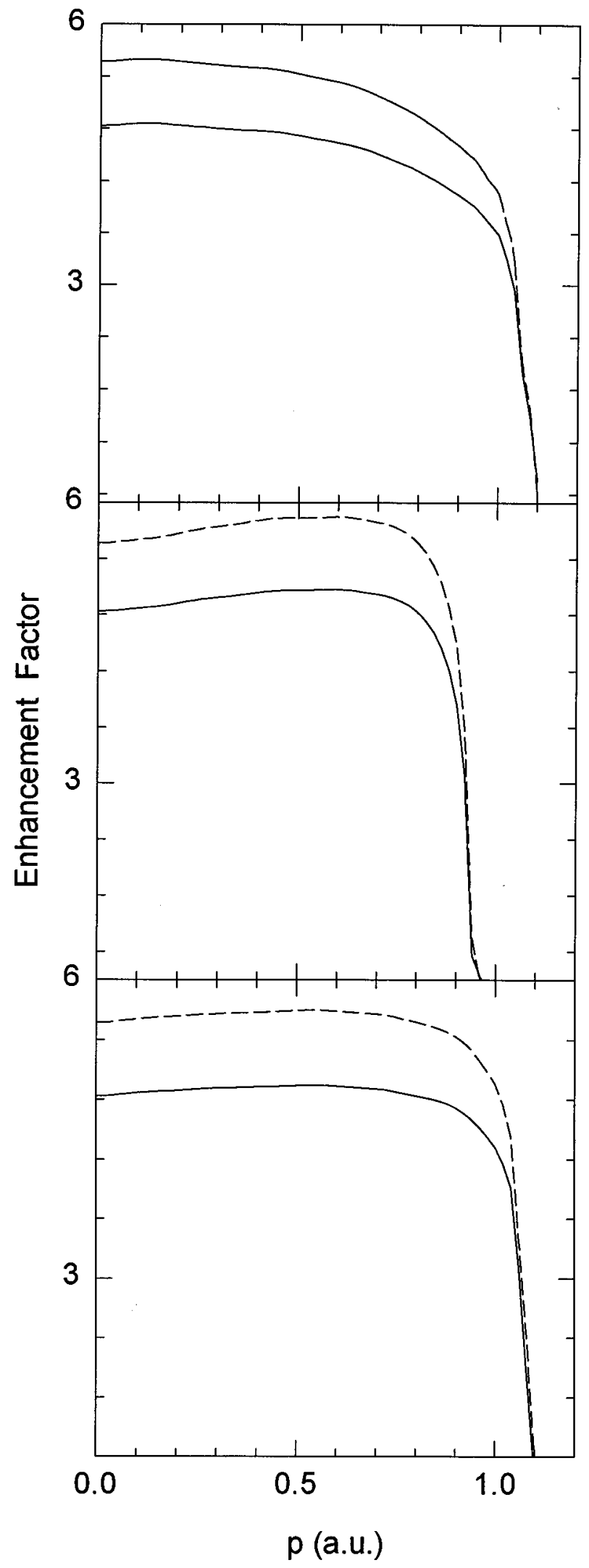

FIG. 4. Enhancement factor along the [100], [110], and [111] directions in $\mathrm{Si}$. The top, middle, and bottom figures correspond to the [100], [110], and [111] directions, respectively. Notations for the GGA and LDA methods are shown by solid and dashed lines, respectively. 
TABLE I. Comparison of positron lifetimes obtained in the $a b$ initio pseudopotentials method with experiment. The lifetime calculated in the LDA and GGA formalisms are denoted as $\tau^{\mathrm{LDA}}$ and $\tau^{\mathrm{GGA}}$, respectively. The corresponding valence lifetimes are denoted by a subscript $v$. The lifetime calculated using Eqs. (2.19) and (2.20) are denoted by a subscript $I$.

\begin{tabular}{|c|c|c|c|c|c|c|c|c|c|}
\hline \multirow[b]{2}{*}{ Material } & \multicolumn{2}{|c|}{ This work } & \multicolumn{2}{|c|}{ This work } & \multicolumn{2}{|c|}{ This work } & \multicolumn{2}{|c|}{ LMTO-ASA } & \multirow[b]{2}{*}{$\begin{array}{l}\text { Expt. } \\
\text { (psec) }\end{array}$} \\
\hline & $\begin{array}{c}\tau^{\mathrm{LDA}} \\
(\mathrm{psec})\end{array}$ & $\begin{array}{c}\tau_{v}^{\mathrm{LDA}} \\
(\mathrm{psec})\end{array}$ & $\begin{array}{c}\tau^{\mathrm{GGA}} \\
(\mathrm{psec})\end{array}$ & $\begin{array}{c}\tau_{v}^{\mathrm{GGA}} \\
(\mathrm{psec})\end{array}$ & $\begin{array}{c}\tau_{I}^{\mathrm{LDA}} \\
(\mathrm{psec})\end{array}$ & $\begin{array}{c}\tau_{I}^{\mathrm{GGA}} \\
(\mathrm{psec})\end{array}$ & $\begin{array}{c}\tau^{\mathrm{LDA}} \\
(\mathrm{psec})\end{array}$ & $\begin{array}{c}\tau^{\mathrm{GGA}} \\
(\mathrm{psec})\end{array}$ & \\
\hline $\mathrm{C}$ & 87 & 88 & 100 & 101 & 87 & 100 & 86 & 96 & 98 \\
\hline $\mathrm{Si}$ & 190 & 194 & 216 & 222 & 188 & 217 & 193 & 210 & 216 \\
\hline $\mathrm{Ge}$ & 198 & 212 & 228 & 244 & 193 & 227 & 191 & 228 & 228 \\
\hline
\end{tabular}

is interesting to observe that our calculation has correctly reproduced the lifetime in $\mathrm{Si}$ whereas both the LMTO-ASA and atomic superposition methods find a lifetime of 210 psec. It is difficult for us to give any reason why the pseudopotential calculation for positron lifetime is more accurate in Si.

The calculated lifetimes in some compound semiconductors are tabulated in Table II. As in elemental semiconductors, the positron lifetimes in compound semiconductors are not significantly different whether calculated taking the total electron density as in Eq. (2.1) or treating the valence and core lifetimes separately as in Eqs. (2.19) and (2.20). We have no idea at present why our LDA calculations in both schemes predict a much higher value than the LMTO-ASA method. In $\mathrm{SiC}$ our calculation shows that the positron lifetime is 145 psec whereas the LMTO-ASA method predicts a value of $139 \mathrm{psec}$ and the experiment is $140 \mathrm{psec}$. In GaAs the lifetime is found to be only 1 psec higher than both the experiment and LMTO-ASA calculation. Jhi and $\mathrm{Ihm}$ have shown that in $\mathrm{GaN}$ the explicit treatment of the gallium $3 d$ electrons as interacting valence states rather than inert core states systematically improves various structural and electronic properties. ${ }^{32}$ We also expect that the $3 d$ electrons in GaAs need to be treated as valence electrons to get better agreement with experiment. Although we have not considered the $3 d$ electrons in the pseudopotential calculation, we have got the lifetime close to the experiment since we have used the experimental lattice constant in our calculation. In InP the agreement between theory and experiment is quite similar to that for GaAs. We also suggest that the inclusion of $4 d$ electrons in the pseudopotential calculation is necessary in this case to obtain a better agreement with experiment. It is surprising to find that in CdTe the positron lifetime is found to be slightly higher than the LMTO-ASA value although we have not treated the $d$ electrons of $\mathrm{Cd}$ as valence electrons. Compared to group III-V semiconductors, the $d$ electrons in group II-VI semiconductors are very important. Since we have used the experimental lattice constant in our calculation we have got this agreement. However, for a better agreement the complete cationic $N$ shell needs to be included in the pseudopotential calculation. ${ }^{33}$

\section{CONCLUSION}

In the present work we have calculated the electronpositron momentum distributions in $\mathrm{Si}$ and the positron lifetime in a few semiconductors using both the LDA and GGA methods. Comparison of the calculated momentum distributions with experiment in $\mathrm{Si}$ shows that only GGA scheme correctly gives the magnitude of the experimental data. The anisotropies between [100] and [110] directions and [100] and [111] directions based on the GGA scheme are in very good agreement with experiment. The present GGA scheme for the electron-positron correlation is found to be successful taking the pseudopotential theory into account. The positron lifetime in the GGA scheme in diamond, $\mathrm{Si}, \mathrm{Ge}, \mathrm{SiC}, \mathrm{GaAs}$, $\mathrm{InP}$, and CdTe are in good agreement with the experiment as in the LMTO-ASA method. Although we have not included

TABLE II. Comparison of lifetimes obtained in the ab initio pseudopotentials method. Notations are the same as in Table I.

\begin{tabular}{|c|c|c|c|c|c|c|c|c|c|}
\hline \multirow[b]{2}{*}{ Material } & \multicolumn{2}{|c|}{ This work } & \multicolumn{2}{|c|}{ This work } & \multicolumn{2}{|c|}{ This work } & \multicolumn{2}{|c|}{ LMTO-ASA } & \multirow[b]{2}{*}{$\begin{array}{l}\text { Expt. } \\
\text { (psec) }\end{array}$} \\
\hline & $\begin{array}{c}\tau^{\mathrm{LDA}} \\
(\mathrm{psec})\end{array}$ & $\begin{array}{c}\tau_{v}^{\mathrm{LDA}} \\
(\mathrm{psec})\end{array}$ & $\begin{array}{c}\tau^{\mathrm{GGA}} \\
(\mathrm{psec})\end{array}$ & $\begin{array}{c}\tau_{v}^{\mathrm{GGA}} \\
(\mathrm{psec})\end{array}$ & $\begin{array}{c}\tau_{I}^{\mathrm{LDA}} \\
(\mathrm{psec})\end{array}$ & $\begin{array}{c}\tau_{I}^{\mathrm{GGA}} \\
(\mathrm{psec})\end{array}$ & $\begin{array}{c}\tau^{\mathrm{LDA}} \\
(\mathrm{psec})\end{array}$ & $\begin{array}{c}\tau^{\mathrm{GGA}} \\
(\mathrm{psec})\end{array}$ & \\
\hline $\mathrm{SiC}$ & 130 & 134 & 145 & 150 & 130 & 145 & 124 & 139 & 140 \\
\hline GaAs & 197 & 220 & 232 & 256 & 202 & 233 & 190 & 231 & 231 \\
\hline $\mathrm{InP}$ & 213 & 256 & 246 & 293 & 207 & 245 & 201 & 248 & 244 \\
\hline $\mathrm{CdTe}$ & 245 & 302 & 292 & 360 & 249 & 305 & 228 & 290 & 285 \\
\hline
\end{tabular}


the $d$ electrons in the valence bands in GaAs, InP, and CdTe, we have got good results because we have used experimental lattice constants in our calculations. The only drawback of the present positron GGA method is that it is semiempirical.
Moreover, the GGA correction is not carried out with the same method as done in the GGA correction for the electron structure calculation. ${ }^{34}$ In future the present method of GGA scheme needs to be improved.
${ }^{1}$ See, for example, Positron Solid State Physics, edited by W. Brandt and A. Dupasquire (North-Holland, Amsterdam, 1993).

${ }^{2}$ M. J. Puska and R. M. Nieminen, Rev. Mod. Phys. 66, 841 (1994).

${ }^{3}$ W. Kohn and L. J. Sham, Phys. Rev. 140, A1133 (1965).

${ }^{4}$ B. Chakraborty and R. W. Siegel, Phys. Rev. B 27, 4535 (1983).

${ }^{5}$ E. Boronski and R. M. Nieminen, Phys. Rev. B 34, 3820 (1986).

${ }^{6}$ H. Rojas, B. Barbiellini, and T. Jarlborg, Mater. Sci. Forum 105110, 799 (1992).

${ }^{7}$ W. Brandt and J. Reinheimer, Phys. Rev. B 2, 3104 (1970).

${ }^{8}$ M. J. Puska, S. Mäkinen, M. Manninen, and R. M. Nieminen, Phys. Rev. B 39, 7666 (1989).

${ }^{9}$ K. O. Jensen, J. Phys.: Condens. Matter 1, 10595 (1989).

${ }^{10}$ B. Barbiellini, M. J. Puska, T. Torsti, and R. M. Nieminen, Phys. Rev. B 51, 7341 (1995).

${ }^{11}$ B. Barbiellini, M. J. Puska, T. Korhonen, A. Harju, T. Torsti, and R. M. Nieminen, Phys. Rev. B 53, 16201 (1996).

${ }^{12}$ See, for example, Progress in Computational Physics of Matter, edited by L. Reatto and F. Manghi (World Scientific, Singapore, 1995).

${ }^{13}$ R. Car and M. Parrinello, Phys. Rev. Lett. 55, 2471 (1985).

${ }^{14}$ G. B. Bachelet, H. S. Greenside, G. A. Baraffe, and M. Schlüter, Phys. Rev. B 24, 4745 (1981).

${ }^{15}$ G. B. Bachelet, D. R. Hamann, and M. Schlüter, Phys. Rev. B 26, 4199 (1982).

${ }^{16}$ X. Gonze, R. Stumpf, and M. Scheffler, Phys. Rev. B 44, 8503 (1991).

${ }^{17}$ J. P. Perdew and A. Zunger, Phys. Rev. B 23, 5048 (1981).

${ }^{18}$ C. Pannetta and A. Baldereschi, in Positron Annihilation, edited by L. Dorikens-Vanpraet, M. Dorikens, and D. Segers (World Scientific, Singapore, 1989), p. 693.

${ }^{19}$ S. Daniuk, M. Sob, and A. Rubaszek, Phys. Rev. B 43, 2580 (1991).

${ }^{20}$ M. Alatalo, B. Barbiellini, M. Hakala, H. Kauppinen, T. Korhonen, M. J. Puska, K. Saarinen, P. Hautojärvi, and R. M. Nieminen, Phys. Rev. B 54, 2397 (1996).

${ }^{21}$ S. G. Louie, S. Froyen, and M. L. Cohen, Phys. Rev. B 26, 1738 (1982).

${ }^{22}$ M. Saito and A. Oshiyama, Phys. Rev. B 53, 7810 (1996).

${ }^{23}$ J. Ihm, A. Zunger, and M. L. Cohen, J. Phys. C 12, 4409 (1979).

${ }^{24}$ M. L. Cohen and J. R. Chelikowsky, Electronic Structure and Optical Properties of Semiconductors, edited by M. Cardona (Springer-Verlag, Berlin, 1988).

${ }^{25}$ D. J. Chadi and M. L. Cohen, Phys. Rev. B 8, 5747 (1973).

${ }^{26}$ E. Clementi and C. Roetti, At. Data Nucl. Data Tables 14, 177 (1974).

${ }^{27}$ K. Fujiwara and T. Hyodo, J. Phys. Soc. Jpn. 35, 1133 (1973).

${ }^{28}$ W. LiMing, B. K. Panda, S. Fung, and C. D. Beling (unpublished).

${ }^{29}$ J. Mader, S. Berko, H. Krakauer, and A. Bansil, Phys. Rev. Lett. 37, 1232 (1976).

${ }^{30}$ J. C. Erskine and J. D. McGervey, Phys. Rev. 151, 615 (1966).

${ }^{31}$ W. Schülke, Phys. Status Solidi B 82, 229 (1977).

${ }^{32}$ S.-H. Jhi and J. Ihm, Phys. Status Solidi B 191, 387 (1995).

${ }^{33}$ M. Rohlfing, P. Krüger, and J. Pollmann, Phys. Rev. Lett. 75, 3489 (1995)

${ }^{34}$ J. A. White and D. M. Bird, Phys. Rev. B 50, 4954 (1994). 